\title{
Factors are not the same for risk of stopping exclusive breast-feeding and introducing different types of liquids and solids in HIV-affected communities in Ghana
}

\author{
Grace S. Marquis ${ }^{1 *}$, Anna Lartey ${ }^{2}$, Rafael Perez-Escamilla ${ }^{3}$, Robert E. Mazur ${ }^{4}$, Lucy Brakohiapa ${ }^{5}$ \\ and Katherine A. Birks ${ }^{1}$ \\ ${ }^{1}$ School of Dietetics and Human Nutrition, McGill University, Ste. Anne-de-Bellevue, Quebec, Canada H9X 3 V9 \\ ${ }^{2}$ Department of Nutrition and Food Science, University of Ghana, Legon, Ghana \\ ${ }^{3}$ Yale School of Public Health, New Haven, CT 06510, USA \\ ${ }^{4}$ Department of Sociology, Iowa State University, Ames, IA 50011, USA \\ ${ }^{5}$ Nutrition Department, Noguchi Memorial Institute for Medical Research, University of Ghana, Legon, Ghana \\ (Submitted 31 August 2015 - Final revision received 17 March 2016 - Accepted 31 March 2016 - First published online 6 May 2016)
}

\section{Abstract}

Exclusive breast-feeding (EBF) for 6 months supports optimal infant growth, health and development. This paper examined whether maternal HIV status was associated with EBF and other infant feeding practices. Pregnant women were enrolled after HIV counselling, and their babies were followed up for up to 1 year. Data on household socio-economics and demographics, maternal characteristics and infants' daily diet were available for 482 infants and their mothers (150 HIV-positive (HIV-P), 170 HIV-negative (HIV-N) and 162 HIV-unknown (HIV-U)). Survival analyses estimated median EBF duration and time to introduction of liquids and foods; hazards ratios (HR) used data from 1-365 and 1-183 d, adjusting for covariates. Logistic regression estimated the probability of EBF for 6 months. Being HIV-P was associated with a shorter EBF duration (139d) compared with HIV-N (163 d) and HIV-U (165 d) $(P=0.004)$. Compared with HIV-N, being HIV-P was associated with about a $40 \%$ higher risk of stopping EBF at any time point (HR 1.39; $95 \%$ CI 1.06, 1.84; $P=0.018$ ) and less than half as likely to complete 6 months of EBF (adjusted OR $0.42 ; 95 \%$ CI $0 \cdot 22,0 \cdot 81 ; P=0 \cdot 01$ ). Being HIV-P tended to be or was associated with a higher risk of introducing non-milk liquids (HR 1.34; $95 \%$ CI 0.98, 1.83; $P=0.068$ ), animal milks (HR 2.37; $95 \%$ CI 1.32, 4.24; $P=0 \cdot 004$ ) and solids (HR 1.56; $95 \%$ CI 1.10, 2.22; $P=0.011$ ) during the first 6 months. Weight-for-age $Z$-score was associated with EBF and introducing formula. Different factors (ethnicity, food insecurity, HIV testing strategy) were associated with the various feeding behaviours, suggesting that diverse interventions are needed to promote optimal infant feeding.

Key words: HIV: Exclusive breast-feeding: Infant diets: Complementary feeding

Mother-to-child transmission (MTCT) accounts for at least $90 \%$ of HIV infections among children; today, an estimated one-half of new infections occur during breast-feeding ${ }^{(1)}$. The exclusive use of formula eliminates the transmission of the virus via breast milk. However, there is a risk of morbidity and mortality from other infections that is associated with formula feeding and other suboptimal infant feeding practices, even in high-income countries $^{(2,3)}$. The health risks during early infancy are highest where ideal conditions do not exist and mixed feeding (breast milk plus other liquids or solids) is practiced ${ }^{(4-6)}$. For infants living in poor communities with limited access to clean water, exclusive breast-feeding (EBF) for the first 6 months of life is recommended, regardless of their or their mother's HIV status ${ }^{(7)}$.
Despite world-wide recommendations, the majority of mothers do not EBF for 6 months ${ }^{(2,8)}$. Reasons for not practicing $\mathrm{EBF}$ are diverse ${ }^{(9)}$ and may include, among others, (i) advice or social pressure to mix-feed ${ }^{(10-12)}$, (ii) perceptions of inadequate milk supply ${ }^{(11-14)}$ or need for water supplementation ${ }^{(10)}$, (iii) societal feeding norms ${ }^{(13,14)}$ and (iv) lack of adequate knowledge on breast-feeding and health ${ }^{(10,13)}$. HIV seropositive women face additional difficulties related to infant feeding. In societies in which breast-feeding is the norm, mixed feeding rather than exclusive formula feeding may be selected because of fear of disclosing their HIV status and being the target of social stigma ${ }^{(10,15)}$, as well as because of conflicting information and lack of adequate support obtained from

Abbreviations: CF, complementary foods; EBF, exclusive breast-feeding; HIV-P, HIV-positive; HIV-N, HIV-negative; HIV-U, HIV-unknown status; HR, hazard ratios; MTCT, mother-to-child transmission; WAZ, weight-for-age $Z$-score.

* Corresponding author: G. S. Marquis, fax +1 514398 7739, email grace.marquis@mcgill.ca 
healthcare providers ${ }^{(12,13)}$. Recognising these challenges, the World Health Assembly endorsed the Comprehensive Implementation Plan on Maternal, Infant and Young Child Nutrition, including a 2025 target to increase the rate of EBF during the first 6 months of life to at least $50 \%$,(16).

Compliance with both EBF recommendations and lifelong anti-retroviral therapy (ART) regimens for infected women reduces MTCT to a level such that elimination of child infections could be a near-term goal ${ }^{(1)}$. However, both optimal feeding and therapy are needed. The coverage of HIV testing and ART to prevent MTCT has increased markedly in Ghana. The estimated percentage of HIV-infected pregnant women who received ART increased from $38 \%$ in 2008 to $>76 \%$ in 2013, although it dropped again in 2014 to $66 \%{ }^{(17)}$. In contrast, national surveys have shown the opposite trend for infant feeding; there was a decline in EBF between 2008 and 2013 (60-53\% for $2-3$ months; $49-36 \%$ for $4-5$ months ${ }^{(18,19)}$. To develop effective interventions that support EBF for HIV sero-positive and sero-negative mothers, it is important to understand in-depth the infant feeding practices and the factors that are associated with $\mathrm{EBF}$ for the recommended full 6 months.

The Research to Improve Infant Nutrition and Growth (RIING) study investigated the pathways by which maternal HIV status influenced infant nutrition and health. The aim of this analysis was to examine whether maternal HIV status was associated with suboptimal breast-feeding and early introduction of liquids and foods that could put the infant at increased risk of viral transmission and illness, as well as to determine other factors that were associated with infant feeding practices.

\section{Methods}

\section{Study site}

This study was conducted in the Yilo Krobo and Manya Krobo districts in the Eastern region of Ghana between 2003 and 2008. The first birth was in 2004. The HIV infection rate in this region in 2003 was $3.7 \%$ ( $4.4 \%$ of women), the highest in the country and considerably higher than the Ghanaian national prevalence of $2 \cdot 2 \%(2 \cdot 7 \% \text { of women })^{(20)}$.

\section{Study design, sample size and participant recruitment}

Detailed descriptions of the methods have previously been published ${ }^{(21-23)}$. The original RIING sample size was based on the outcome of interest requiring the largest sample size, morbidity (using a one-tailed test, a significance level of $0 \cdot 05$, power of $80 \%$, morbidity rate of $20 \%$ and mean group difference of $15 \%)^{(22)}$. Assuming a loss to follow-up of $25 \%$, the sample size required was 189/group for HIV-positive (HIV-P), HIV-negative (HIV-N) and HIV-unknown (HIV-U) status. The sample available for the present analysis provided a statistical power of $93.1 \%$ for the outcome: HIV group comparison of 6-month EBF rates.

To examine the relationship between maternal HIV status and infant feeding outcomes, the study used a prospective cohort design to follow up caregivers and their infants for 12 months postpartum. Participant recruitment was carried out through the voluntary counselling and testing (VCT) programme of prenatal clinics in the three area hospitals. Women were counselled on the risk of HIV transmission, informed about the services available to them and, until 2005 through the 'opt-in' system, were invited to be tested for $\mathrm{HIV}^{(21)}$. After 2005, the national programme changed to an 'opt-out' structure, in which all women were tested unless they specifically requested otherwise. Once post-test counselling had occurred or mothers refused to be tested (HIV-U), the hospital VCT nurse informed the women of the study and identified those who were interested in participating. Project staff then obtained written informed consent from women for their participation and that of their infant.

Eligibility criteria included that the mother be pregnant at the time of enrolment, be at least 18 years of age, participate in VCT pre-test counselling, agree to have her HIV status released to the project supervisor if tested, plan to be available for the entire duration of the study and have no clinical or physical ailments that would limit her ability to care for her infant. In addition, mothers had to give birth to a live infant free of birth defects that might impair feeding. Only data from the second registered twin were included.

As part of the national protocol for the prevention of MTCT, nevirapine was provided by the hospitals to all women during labour and to the infant at birth ${ }^{(21)}$. Ethics approval for the RIING study was obtained from the Institutional Review Boards of the participating research institutions: University of Ghana, Iowa State University, University of Connecticut and McGill University.

\section{Data collection}

Maternal HIV status was determined by the hospital staff, using the Rapid Test Abbott Determine HIV-1/2 (Abbott Laboratories). Only survey variables used in the analyses are described here. These included the following: enrolment household socioeconomic and demographic data and maternal obstetric history; household food security measured with an experiential-based eight-item survey that was adapted from the US Household Food Security Survey Model ${ }^{(23,24)}$ and used at birth, and 3, 6, 9 and 12 months; maternal postnatal depression measured at birth and 6 and 12 months using the ten-item Edinburgh Postnatal Depression Scale ${ }^{(25)}$; infant birth weight when the birth occurred in a health facility; and daily breast-feeding and complementary feeding practices evaluated based on World Health Organization definitions ${ }^{(26)}$. Mothers were visited at home twice weekly and were asked about their infant's intake of breast milk, non-milk liquids, animal-based (non-human) milks, infant formula and solid and semi-solid foods, as well as symptoms of illness ( $\geq 3$ liquid stools/d (diarrhoea), fever, cough and/or difficulty breathing (acute respiratory infection)) during the previous days since the last visit ${ }^{(21)}$. Weight to the nearest $100 \mathrm{~g}$ (Tanita Corporation of America Inc.) and length to the nearest $0 \cdot 1 \mathrm{~cm}$ (Shorr Productions) were measured monthly ${ }^{(22)}$. Results from data on morbidity and growth measurements have been previously published ${ }^{(21,22)}$. 


\section{Data analysis}

Analyses were performed using SAS version 9.3 (SAS Institute). A food insecurity score was constructed with seven of the eight items (one item was considered redundant), using Rasch modelling to determine the psychometric validity of the scale items ${ }^{(23)}$. Households were initially categorised as food secure (0 affirmative responses), mild/moderately food insecure (1-4 affirmative responses) and severely food insecure (5-7 affirmative responses). Final models included households as either food secure (0) or food insecure (1-7). A household amenities score was created as a proxy for socio-economic status, and was developed using eighteen variables related to a household's socio-economic status (household materials, household water, access to electricity and cooking fuel, appliance ownership) ${ }^{(22)}$. Factor analysis with varimax rotation was used to calculate the final score for each household, with a lower value indicating a poorer household. A dichotomous variable was developed from the postnatal depression scale, with $<13$ categorised as not showing symptoms (no) and 13 and above as showing symptoms (yes) ${ }^{(21)}$. A birth-related variable was defined to reflect the opt-in/opt-out HIV testing system in the Ghana Health Services (2004/2005=opt-in, 2006 and beyond =opt-out). A dichotomous illness variable was calculated if the child had at least one symptom (diarrhoea, fever, acute respiratory infection) in the past $30 \mathrm{~d}$. Weight was converted to weight-for-age $Z$-score (WAZ) using the WHO Child Growth Standards ${ }^{(27)}$. The World Health Organization ${ }^{(28)}$ guidelines for breast-feeding among HIV-infected women changed during the study. Birth date was also dichotomised as before $(<2007)$ and after $(\geq 2007)$ to capture the differences in policy.

Descriptive analyses compared characteristics at enrolment (or at birth where indicated) by maternal HIV status using ANOVA (with Bonferroni post hoc tests) or $\chi^{2}$ tests as appropriate, and between mothers who EBF for 6 months or longer and those who breast-fed for $<6$ months using independent Student's $t$ tests or $\chi^{2}$ tests, as appropriate. Kaplan-Meier curves were used to calculate and compare across HIV groups the median survival times in days for time to cessation of EBF, and time to introduction of non-milk liquids, animal-based (non-human) milks, and solids and semi-solids. In the case of formula, a median survival time could not be estimated; the 25 th percentile was presented. Censoring was indicated if the mother was still EBF or had not yet introduced a liquid or food category on the last day of data collection. If data were missing for more than $30 \mathrm{~d}$, the last day of valid data before the gap was counted and the mother's data were then censored. The logrank (Mantel-Cox) test of equality of survival distribution was used to determine significant differences between HIV groups for time to cessation of EBF and time of introduction of each liquid or food category. The Šidák correction was used to compare differences across HIV groups. The percentage of mothers who were breast-feeding at 6,9 and 12 months was compared across HIV groups using $\chi^{2}$ tests.

Bivariate survival analyses using Cox regression were performed between socio-demographic variables (at enrolment or at birth) and time to cessation of EBF, as well as time of introduction of formula, non-milk liquids, animal-based milks and solids and semi-solids. Food insecurity at birth, 3, 6, 9 and 12 months; symptoms of postnatal depression at birth, 6 and 12 months; and monthly morbidity and anthropometric indicators were included as time-dependent covariates.

Multi-variable survival analyses were conducted to determine predictors of time to cessation of EBF and time to introduction of each liquid and food category, using all available data between birth and $365 \mathrm{~d}$. Independent variables were initially included in the models if $P<0.25$ in the bivariate analyses; after backward elimination was performed, variables with $P<0.10$ in at least one of the models were kept in all final models. A mother's HIV status, age, ethnicity, marital status, household amenities, the number of children under 5 years of age, household food insecurity, opt-in/out-out system, morbidity and WAZ remained in the final models. Hazard ratios (HR) were calculated for each independent variable and reflect risk of outcome at any point in time from birth to $365 \mathrm{~d}$. The models were rerun to estimate HR for time to introduction of each complementary liquid and food solely for the period $0-183 \mathrm{~d}$, the period during which EBF is recommended. A similar 0-183-d model for EBF was not included, as all mothers had ended EBF by the 7th month and the results were essentially identical to that of the $0-365-\mathrm{d}$ model included here.

A bivariate logistic regression was conducted to determine the factors associated with the dichotomous variable of EBF for 6 months or longer. In the multi-variable logistic regression, the same independent variables were initially included in the model if $P<0.25$ in bivariate analyses. Backward elimination was performed and variables with $P<0 \cdot 10$ were kept in the final model. A mother's HIV status, ethnicity and the number of children under 5 years of age remained in the final model.

Finally, to assess the possible influence of the change in the WHO guidelines on infant feeding recommendations, weaning prevalence was compared by birth date $(<2007 v$. $\geq 2007)$ and HIV status using the Cochran-Mantel-Haenszel test with the continuity correction, from 7 to 12 months. All statistically significant associations are reported with at least a $P<0 \cdot 05$.

\section{Results}

\section{Study population}

A total of 552 women were enrolled in the study and 503 women had a live birth (including twelve sets of twins). Data from 482 infants and their mothers (150 HIV-P, 170 HIV-N and 162 HIV-U) were available for this analysis. During the 12 months of follow-up, fourteen moved out of the study area (five HIV-P, two HIV-N and seven HIV-U), forty-six withdrew from the study (twenty-five HIV-P, eleven HIV-N and ten HIV-U), ten mothers or children died (five HIV-P, one HIV-N and four HIV-U), five HIV-P mothers withdrew because of illness of mother or child and the field work finished before data collection was completed with eleven mothers and their children (seven HIV-P, two HIV-N and two HIV-U), giving a group difference in length of follow-up $(P<0 \cdot 001)$. All 482 participants were included in the study's analysis up to the 
last day of available information; censoring was indicated, where necessary.

\section{Maternal and household characteristics}

Maternal age ranged from 18 to 50 years with a mean age of 28.4 (SD 5.9) years. Approximately half of mothers $(56.4 \%$, $n$ 272) were traders or earned a salary or wages, one-third of the mothers $(33.2 \%, n 160)$ were artisans or students, $2.3 \%$ ( $n$ 11) were farmers and $8.1 \%$ ( $n$ 39) were unemployed. The majority of women $(68.5 \%, n 330)$ were of the Ga/Adangbe ethnic group; the proportion was highest among the HIV-P mothers (Table 1). Several socio-economic indicators suggested a more impoverished status among HIV-P mothers than those in the HIV-N and HIV-U groups, including having less education, fewer household amenities and less likely to live with a partner/husband.

\section{Infant characteristics and feeding practices}

The mean infant birth weight was about $200 \mathrm{~g}$ lower among the HIV-P group compared with the other groups $(P=0 \cdot 006)$, with no

Table 1. Characteristics of Ghanaian mother-infant pairs at enrolment, by maternal HIV status (Numbers and percentages; mean values and standard deviations)

\begin{tabular}{|c|c|c|c|c|c|c|c|}
\hline \multirow[b]{3}{*}{ Characteristics } & \multicolumn{7}{|c|}{ Maternal HIV status } \\
\hline & \multicolumn{2}{|c|}{ HIV-P } & \multicolumn{2}{|c|}{ HIV-N } & \multicolumn{2}{|c|}{ HIV-U } & \multirow[b]{2}{*}{$P$} \\
\hline & $n$ & $\%$ & $n$ & $\%$ & $n$ & $\%$ & \\
\hline \multicolumn{8}{|l|}{ Mother } \\
\hline Age (years) & 150 & & 170 & & 162 & & 0.377 \\
\hline Mean & \multicolumn{2}{|c|}{$28 \cdot 2$} & \multicolumn{2}{|c|}{28.9} & \multicolumn{2}{|c|}{$28 \cdot 0$} & \\
\hline SD & \multicolumn{2}{|c|}{$5 \cdot 8$} & \multicolumn{2}{|c|}{$6 \cdot 0$} & \multicolumn{2}{|c|}{$5 \cdot 7$} & \\
\hline Live births $(n)$ & 150 & & 170 & & 162 & & 0.496 \\
\hline Mean & \multicolumn{2}{|c|}{1.5} & \multicolumn{2}{|c|}{$1 \cdot 6$} & \multicolumn{2}{|c|}{1.4} & \\
\hline SD & \multicolumn{2}{|c|}{1.5} & \multicolumn{2}{|c|}{1.5} & \multicolumn{2}{|c|}{1.4} & \\
\hline \multicolumn{7}{|l|}{ Formal education } & 0.002 \\
\hline None & 24 & $16 \cdot 0$ & 12 & $7 \cdot 1$ & 14 & $8 \cdot 6$ & \\
\hline Some primary & 24 & $16 \cdot 0$ & 13 & $7 \cdot 7$ & 16 & 9.9 & \\
\hline Completed primary & 36 & $24 \cdot 0$ & 32 & $18 \cdot 8$ & 41 & $25 \cdot 3$ & \\
\hline Completed junior/secondary or above & 66 & $44 \cdot 0$ & 113 & $66 \cdot 5$ & 91 & $56 \cdot 2$ & \\
\hline \multicolumn{7}{|l|}{ Ethnicity } & 0.0001 \\
\hline Ga/Adangbe & 125 & $83 \cdot 3$ & 108 & $63 \cdot 5$ & 97 & $59 \cdot 9$ & \\
\hline Ewe & 17 & $11 \cdot 3$ & 40 & $23 \cdot 5$ & 41 & $25 \cdot 3$ & \\
\hline Other* & 8 & $5 \cdot 3$ & 22 & $12 \cdot 9$ & 24 & $14 \cdot 8$ & \\
\hline \multicolumn{7}{|l|}{ Marital status } & 0.005 \\
\hline Living with partner/husband & 108 & $72 \cdot 0$ & 146 & 85.9 & 134 & $82 \cdot 7$ & \\
\hline Other† & 42 & $28 \cdot 0$ & 24 & $14 \cdot 1$ & 28 & $17 \cdot 3$ & \\
\hline \multicolumn{7}{|l|}{ Postnatal depression symptoms at birth $\ddagger$} & 0.079 \\
\hline No & 120 & $85 \cdot 1$ & 152 & $92 \cdot 7$ & 140 & $90 \cdot 9$ & \\
\hline Yes & 21 & $14 \cdot 9$ & 12 & $7 \cdot 3$ & 14 & $9 \cdot 1$ & \\
\hline \multicolumn{8}{|l|}{ Household } \\
\hline Food insecurity§ & & & & & & & 0.935 \\
\hline Food secure & 77 & $62 \cdot 1$ & 88 & $64 \cdot 2$ & 86 & $62 \cdot 8$ & \\
\hline Food insecure & 47 & $37 \cdot 9$ & 49 & $35 \cdot 8$ & 51 & $37 \cdot 2$ & \\
\hline Amenities scorell & 150 & & 170 & & 162 & & $<0.0001$ \\
\hline Mean & & & & & & & \\
\hline SD & & & & & & & \\
\hline Children $<5$ years in house $(n)$ & 150 & & 170 & & 162 & & 0.208 \\
\hline Mean & & & & & & & \\
\hline SD & & & & & & & \\
\hline Infant & & & & & & & \\
\hline Sex & & & & & & & 0.873 \\
\hline Male & 77 & $52 \cdot 0$ & 88 & $51 \cdot 8$ & 80 & $49 \cdot 4$ & \\
\hline Female & 71 & $48 \cdot 0$ & 82 & $48 \cdot 2$ & 82 & $50 \cdot 6$ & \\
\hline Weight at birth $(\mathrm{kg})$ & 150 & & 170 & & 162 & & 0.006 \\
\hline Mean & & & & & & & \\
\hline SD & & & & & & & \\
\hline
\end{tabular}

HIV-P, HIV-positive; HIV-N, HIV-negative; HIV-U, HIV-unknown status.

a,b Mean values within a row with unlike superscript letters were significantly different $(P<0.05)$.

* Akan, northern groups.

† Widowed, divorced or single.

$\ddagger$ A dichotomous variable was developed from the Edinburgh Postnatal Depression Scale ${ }^{(25)} ;<13$ categorised as not showing symptoms (no) and $\geq 13$ as showing symptoms (yes).

$\S$ Food insecurity score was created from an eight-item household food security survey adapted from the US Household Food Security Survey Model $^{(23,24)}$; food secure (0 positive responses), food insecure ( $\geq 1$ positive responses).

II Household amenities score was created from a set of eighteen socio-economic variables (household building material, access to water, electricity and ownership of appliances) using factor analysis with varimax rotation; lower values reflect poorer status ${ }^{(22)}$. 
difference in sex (Table 1). The median duration of EBF was $158 \mathrm{~d}$ (approximately 5.3 months), with the longest at $211 \mathrm{~d}$. Being HIV-P was associated with a significantly shorter duration of EBF ( $P<0.01$; Table 2, Fig. 1): a difference of $24 \mathrm{~d}$ compared with HIV-N mothers and $26 \mathrm{~d}$ compared with HIV-U mothers. A higher percentage of HIV-N $(22.9 \%, n$ 39) and HIV-U (22.2\%, $n$ 36) compared with HIV-P $(10.0 \%, n$ 15) mothers EBF for at least 6 months $(P=0.005)$. In addition, a higher percentage of mothers from the $\mathrm{Ga} /$ Adangbe $(18.2 \%, n 60 / 330)$ and Ewe $(25.5 \%$, $n$ 25/98) ethnic groups EBF for at least 6 months compared with mothers of the 'other' ethnic group ( $9.3 \%, n 5 / 54 ; P=0.045)$. The number of children under 5 years of age tended to be higher in households in which mothers EBF for at least 6 months compared with mothers who did not do so (1.5 (SD 0.7) v. 1.4 (SD 0.6); $P=0.08$ ). Other variables were not associated with EBF for at least 6 months. The association between breast-feeding and HIV continued after EBF ended. Compared with HIV-P mothers, a higher percentage of HIV-N and HIV-U mothers were breast-feeding at 6 , 9 and 12 months (all $P<0 \cdot 0001$; Table 2).

Mixed feeding was common throughout the first 6 months, ranging from $10 \%$ in the 1 st month to $76 \%$ at 6 months (Table 2). There was no statistically significant group difference in mixed feeding per se; however, groups did differ by what was given. In the bivariate analyses, non-milk liquids were introduced earlier among HIV-P mothers compared with HIV-N (median survival differences of $27 \mathrm{~d} ; P=0.003$; Fig. 1); the difference of $26 \mathrm{~d}$ compared with HIV-U did not reach significance $(P=0.08)$. HIV-P mothers introduced animal milks $49 \mathrm{~d}$ earlier than HIV-N mothers $(P=0.037)$, but timing was similar to that of HIV-U mothers. There was no significant difference by HIV status in the age of introduction of semi-solids/solids, with the median in the three groups between 5 and 6 months. Infant formula was uncommon and used by five HIV-P, eight HIV-N and seven HIV-U mothers in the 1st month; this increased to twenty-six HIV-P, twenty-four HIV-N and twenty HIV-U mothers, cumulatively, by 4 months of age. HIV-U mothers tended to introduce formula earlier compared with HIV-P mothers $(P=0 \cdot 080)$; no other differences were noted.

\section{Survival analysis models}

The initial multi-variable Cox regression analyses reflected risk of outcome at all time points throughout the 1st year and demonstrated that different factors were associated with the various infant feeding practices (Table 3). The risk of stopping EBF was higher among HIV-P and those of an 'other' ethnic

Table 2. Infant feeding characteristics and time of introduction of complementary foods for Ghanaian infants, by maternal HIV status (Numbers and percentages; medians and $95 \%$ confidence intervals)

\begin{tabular}{|c|c|c|c|c|c|c|c|}
\hline & \multicolumn{6}{|c|}{ Maternal HIV status } & \multirow[b]{3}{*}{$P^{\star}$} \\
\hline & \multicolumn{2}{|c|}{ HIV-P } & \multicolumn{2}{|c|}{ HIV-N } & \multicolumn{2}{|c|}{ HIV-U } & \\
\hline & $n$ & $\%$ & $n$ & $\%$ & $n$ & $\%$ & \\
\hline Exclusive breast-feeding duration (d) & 150 & & 170 & & 162 & & 0.004 \\
\hline Median & \multicolumn{2}{|c|}{$139^{a}$} & \multicolumn{2}{|c|}{$163^{b}$} & \multicolumn{2}{|c|}{$165^{\mathrm{b}}$} & \\
\hline $95 \% \mathrm{Cl}$ & \multicolumn{2}{|c|}{118,152} & \multicolumn{2}{|c|}{155,174} & \multicolumn{2}{|c|}{154,170} & \\
\hline Any breast-feeding (yes) & \multicolumn{2}{|c|}{ 103-120† } & \multicolumn{2}{|c|}{$152-159$} & \multicolumn{2}{|c|}{$141-150$} & \\
\hline At 6 months & 108 & $90 \cdot 0$ & 159 & $100 \cdot 0$ & 150 & $100 \cdot 0$ & $<0.0001$ \\
\hline At 9 months & 89 & $84 \cdot 8$ & 156 & $99 \cdot 4$ & 145 & $99 \cdot 3$ & $<0.0001$ \\
\hline At 12 months & 76 & 73.8 & 146 & $96 \cdot 1$ & 134 & $95 \cdot 0$ & $<0.0001$ \\
\hline Mixed feeding (yes)‡ & $120-150$ & & $159-170$ & & $150-16$ & & \\
\hline At 1 months & 17 & $11 \cdot 3$ & 19 & $11 \cdot 2$ & 10 & $06 \cdot 2$ & 0.201 \\
\hline At 2 months & 25 & $18 \cdot 0$ & 30 & $18 \cdot 3$ & 19 & 11.9 & 0.215 \\
\hline At 3 months & 42 & 31.6 & 35 & 21.5 & 34 & 21.5 & 0.075 \\
\hline At 4 months & 49 & 38.0 & 48 & $30 \cdot 0$ & 46 & $29 \cdot 7$ & 0.249 \\
\hline At 5 months & 65 & $51 \cdot 2$ & 62 & $39 \cdot 0$ & 66 & 42.9 & 0.113 \\
\hline At 6 months & 93 & 77.5 & 120 & $75 \cdot 5$ & 114 & $76 \cdot 0$ & 0.922 \\
\hline \multicolumn{8}{|c|}{ Timing of introduction of complementary feeding } \\
\hline Formula (d) & 150 & & 170 & & 162 & & $0 \cdot 103$ \\
\hline Median & \multirow{2}{*}{\multicolumn{2}{|c|}{$\begin{array}{c}182 \\
142,304\end{array}$}} & \multirow{2}{*}{\multicolumn{2}{|c|}{$\begin{array}{c}187 \\
173,234\end{array}$}} & \multicolumn{2}{|c|}{$285 \S$} & \\
\hline $95 \% \mathrm{Cl}$ & & & & & \multicolumn{2}{|c|}{$188,-$} & \\
\hline Non-milk liquids (d) & \multicolumn{2}{|l|}{150} & \multicolumn{2}{|l|}{170} & 162 & & 0.004 \\
\hline Median & \multicolumn{2}{|c|}{$140^{\mathrm{a}}$} & \multicolumn{2}{|c|}{$166^{\mathrm{a}, \mathrm{b}}$} & \multicolumn{2}{|c|}{$167^{\mathrm{b}}$} & \\
\hline $95 \% \mathrm{Cl}$ & \multicolumn{2}{|c|}{118,155} & \multicolumn{2}{|c|}{157,176} & \multicolumn{2}{|c|}{155,172} & \\
\hline Animal milks (d) & 150 & & 170 & & 162 & & 0.010 \\
\hline Median & \multicolumn{2}{|c|}{$214^{a}$} & \multicolumn{2}{|c|}{$263^{b}$} & \multicolumn{2}{|c|}{$201^{\mathrm{a}}$} & \\
\hline $95 \% \mathrm{Cl}$ & & & & & & & \\
\hline Solids/semi-solids (d) & 150 & & 170 & & 162 & & 0.173 \\
\hline Median & & & & & & & \\
\hline $95 \% \mathrm{Cl}$ & & & & & & & \\
\hline
\end{tabular}

HIV-P, HIV-positive; HIV-N, HIV-negative; HIV-U, HIV-unknown status.

a,b Median values within a row with unlike superscript letters were significantly different $(P<0.05)$

* HIV group difference tested with log-rank (Mantel-Cox) test of equality of survival distribution; Šidák correction was used to compare differences across groups.

$\dagger$ Range in $n$ for the indicated age and HIV status.

¥ Mixed feeding: breast-feeding plus any type of non-human milk liquids and/or solids/semisolids.

$\S$ Median could not be calculated for formula; values represent Q1 (lower 95\% Cl only could be estimated). 
(a)

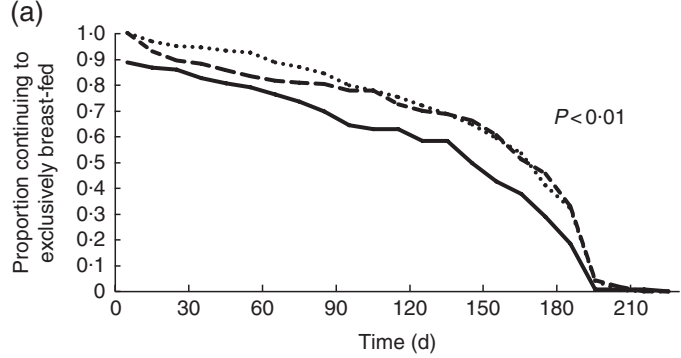

(c)

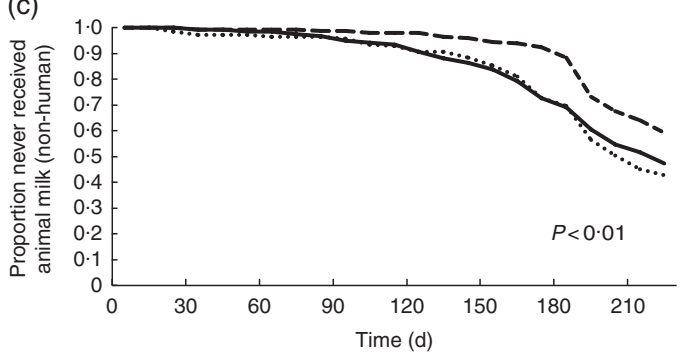

(b)

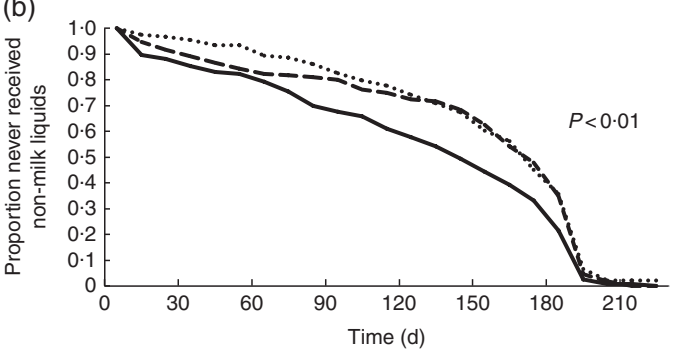

(d)

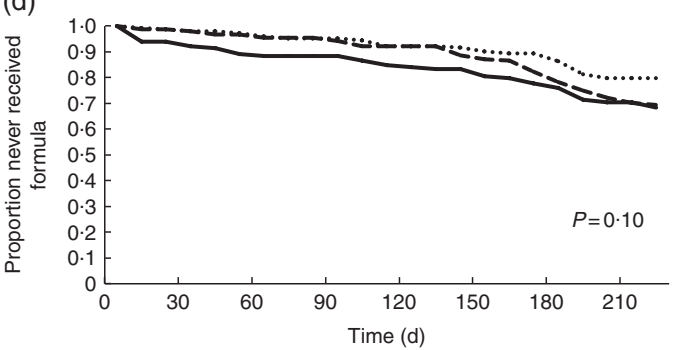

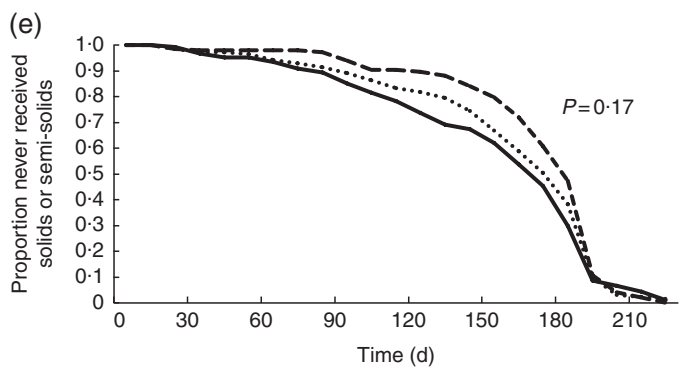

Fig. 1. Bivariate survival analysis results for 478 Ghanaian women: proportion continuing to exclusively breast-feed (a), and not introducing non-milk liquids (b), animal milks (non-human) (c), formula (d) and solids/semi-solids (e), by HIV status ( , positive; - - - -, negative; , unknown)

group (about half were northern), and was lower in households with more young children and among infants with better WAZ. Compared with being HIV-N, an HIV-P status was associated with about a $40 \%$ increased risk of stopping EBF at any point of time. In the bivariate survival analysis for EBF, the three ethnicity curves diverged at about 4 months of age.

Having a higher number of amenities and having a lower WAZ were associated with an increased risk of introducing formula. Risk of introducing formula tended to be lower in the HIV-U group $(P=0.059)$ and among those who were food insecure $(P=0.077)$. Risk tended to be higher among those living alone $(P=0.055)$ and whose children were sick over the past $30 \mathrm{~d}(P=0.095)$. The difference by marital status was noted in the bivariate survival analysis from the 1st month of life. When the analysis was repeated separately for only the 0- to 6-month period ( $n$ 478), the risk was still reduced among the HIV-U group (HR 0.55; $95 \%$ CI $0.31,0 \cdot 98 ; P=0.044$ ).

The number of young children in the home, but not HIV status, was negatively associated in the full-year model with a risk of introduction of non-milk liquids. Maternal age $(P=0.055)$ and WAZ $(P=0.073)$ tended to be associated with a lower risk of use of non-milk liquids. However, when the analysis was done separately for only 0-6 months, HIV-P status tended to be associated with a higher risk of introduction of non-milk liquids (HR 1.34; $95 \%$ CI 0.98, 1.83; $P=0.068$ ), and the association with WAZ became significant (HR 0.87; $95 \%$ CI $0.78,0.96 ; P=0.007)$.

The risk of introducing animal milks over the entire 12 months was higher among HIV-P and HIV-U compared with HIV-N mothers in addition to those households with more amenities. However, mothers who enrolled during the opt-out period were $>40 \%$ less likely to introduce animal milks compared with those who were enrolled earlier. Bivariate survival curves demonstrated a rapid introduction of animal milks in the 6th month among those who were enrolled when the opt-in system was in place; similar behaviour was not seen among participants who were part of the opt-out system (data not shown). Results from only the first 6 months showed an even stronger association with a more than 2-fold higher risk of introducing animal milks with HIV-P and HIV-U status (HR 2.37; $95 \%$ CI $1.32,4.24 ; P=0.004$ and HR $2 \cdot 11 ; 95 \%$ CI $1.23,3.64$; $P<0 \cdot 007$, respectively).

The risk of introduction of solids and semi-solids was reduced with having a larger number of children in the home and with household food insecurity. Higher WAZ tended to be associated with a lower risk, whereas illness was associated with a higher risk of introducing solids. When the model was rerun for 0-6 months only, all associations remained similar except for HIV, ethnicity and WAZ. During the first 6 months, HIV-P was strongly associated with an increased risk of 
Table 3. Multivariable survival analysis for factors associated with time to stopping exclusive breast-feeding and time to introducing formula, non-milk liquids, animal milks and solids/semi-solids among Ghanaian mothers

(Hazards ratios (HR) and $95 \%$ confidence intervals)

\begin{tabular}{|c|c|c|c|c|c|c|c|c|c|c|c|c|c|c|c|}
\hline \multirow[b]{3}{*}{ Factors } & \multicolumn{15}{|c|}{ Infant feeding outcomes during $1-365 d$} \\
\hline & \multicolumn{3}{|c|}{$\begin{array}{l}\text { Stopping exclusive } \\
\text { breast-feeding ( } n \text { 478) }\end{array}$} & \multicolumn{3}{|c|}{$\begin{array}{c}\text { Introducing } \\
\text { formula ( } n \text { 478) }\end{array}$} & \multicolumn{3}{|c|}{$\begin{array}{l}\text { Introducing non-milk } \\
\text { liquids ( } n \text { 478) }\end{array}$} & \multicolumn{3}{|c|}{$\begin{array}{l}\text { Introducing animal } \\
\text { milks ( } n \text { 478) }\end{array}$} & \multicolumn{3}{|c|}{$\begin{array}{c}\text { Introducing } \\
\text { solids/semi-solids ( } n \text { 478) }\end{array}$} \\
\hline & $\mathrm{HR}$ & $95 \% \mathrm{Cl}$ & $P$ & $\mathrm{HR}$ & $95 \% \mathrm{Cl}$ & $P$ & $\mathrm{HR}$ & $95 \% \mathrm{Cl}$ & $P$ & $\mathrm{HR}$ & $95 \% \mathrm{Cl}$ & $P$ & $\mathrm{HR}$ & $95 \% \mathrm{Cl}$ & $P$ \\
\hline \multicolumn{16}{|l|}{ Maternal } \\
\hline \multicolumn{16}{|l|}{ HIV status } \\
\hline Negative & 1 & & & 1 & & & 1 & & & 1 & & & 1 & & \\
\hline Positive & 1.39 & $1.06,1.84$ & 0.018 & 1.04 & $0.63,1.74$ & 0.873 & 1.23 & $0.93,1.63$ & 0.144 & 1.43 & $1.01,2.03$ & 0.045 & 1.21 & $0.90,1.62$ & 0.203 \\
\hline Unknown & 1.03 & $0.81,1.31$ & 0.784 & 0.65 & $0.41,1.02$ & 0.059 & 0.96 & $0.75,1.22$ & 0.733 & 1.49 & $1.11,2.02$ & 0.009 & $1 \cdot 16$ & $0.90,1.49$ & 0.243 \\
\hline Age (years) & 0.99 & $0.97,1.01$ & 0.262 & 1.02 & $0.98,1.05$ & 0.346 & 0.98 & $0.96,1.00$ & 0.055 & 0.99 & $0.97,1.01$ & 0.357 & 1.00 & $0.98,1.02$ & 0.717 \\
\hline \multicolumn{16}{|l|}{ Ethnicity } \\
\hline Ga/Adangbe & 1 & & & 1 & & & 1 & & & 1 & & & 1 & & \\
\hline Ewe & 0.88 & $0.69,1.14$ & 0.334 & 0.95 & $0.60,1.52$ & 0.835 & 0.91 & $0.70,1 \cdot 17$ & 0.447 & 0.87 & $0.63,1.20$ & 0.387 & 0.91 & $0.70,1.18$ & 0.482 \\
\hline Other & 1.49 & $1 \cdot 06,2 \cdot 10$ & 0.021 & 0.96 & $0.51,1.80$ & 0.894 & $1 \cdot 18$ & $0.83,1.67$ & 0.362 & 0.80 & $0.53,1.23$ & 0.310 & 1.34 & $0.94,1.92$ & 0.106 \\
\hline \multicolumn{16}{|l|}{ Marital status } \\
\hline Living with husband/partner & 1 & & & 1 & & & 1 & & & 1 & & & 1 & & \\
\hline Living alone† & 1.02 & $0.78,1.33$ & 0.895 & 1.59 & $0.99,2.56$ & 0.054 & $1 \cdot 11$ & $0.84,1.45$ & 0.474 & 0.89 & $0.63,1.26$ & 0.499 & 0.91 & $0.68,1.22$ & 0.530 \\
\hline \multicolumn{16}{|l|}{ Household } \\
\hline Amenities $(n) \ddagger$ & 0.98 & $0.86,1 \cdot 12$ & 0.814 & 1.60 & $1.27,2.02$ & $<0.0001$ & 0.95 & $0.83,1.09$ & 0.472 & 1.19 & $1.01,1.39$ & 0.034 & 0.92 & $0.80,1.05$ & 0.208 \\
\hline Children $<5$ years $(n)$ & 0.83 & $0.70,0.97$ & 0.022 & 0.87 & $0.64,1.20$ & 0.404 & 0.81 & $0.68,0.96$ & 0.015 & $1 \cdot 13$ & $0.92,1.39$ & 0.258 & 0.73 & $0.61,0.88$ & 0.001 \\
\hline \multicolumn{16}{|l|}{ Food insecurity§ } \\
\hline No & 1 & & & 1 & & & 1 & & & 1 & & & 1 & & \\
\hline Yes & 0.90 & $0.72,1 \cdot 14$ & 0.388 & 0.66 & $0.42,1.05$ & 0.077 & 0.88 & $0 \cdot 70,1 \cdot 12$ & 0.298 & 0.87 & $0.64,1 \cdot 17$ & 0.340 & 0.69 & $0.54,0.89$ & 0.005 \\
\hline \multicolumn{16}{|l|}{ Yearll } \\
\hline 2004/2005 (opt-in) & 1 & & & 1 & & & 1 & & & 1 & & & 1 & & \\
\hline 2006-2008 (opt-out) & 0.97 & $0.78,1.19$ & 0.758 & 0.73 & $0.50,1.08$ & 0.116 & 1.00 & $0.81,1.24$ & 0.999 & 0.58 & $0.44,0.75$ & $<0.0001$ & 0.92 & $0.73,1.15$ & 0.451 \\
\hline Weight-for-age $Z$-score & 0.90 & $0.83,0.98$ & 0.018 & 0.81 & $0.68,0.96$ & 0.013 & 0.92 & $0.85,1.01$ & 0.073 & 0.95 & $0.85,1.05$ & 0.315 & 0.91 & $0.83,1.00$ & 0.055 \\
\hline \multicolumn{16}{|l|}{ Illnessף } \\
\hline No & 1 & & & 1 & & & 1 & & & 1 & & & 1 & & \\
\hline Yes & 1.00 & $0.81,1.24$ & 0.994 & 1.41 & $0.94,2.13$ & 0.095 & 1.05 & $0.84,1 \cdot 30$ & 0.690 & $1 \cdot 11$ & $0.85,1.44$ & 0.441 & 1.24 & $0.99,1.55$ & 0.067 \\
\hline
\end{tabular}

* Akan, northern groups.

† Widowed, divorced or single.

¥ Household amenities score was created from a set of eighteen socio-economic variables (household building material, access to water, electricity and ownership of appliances) using factor analysis with varimax rotation. It is a continuous variable in which lower values reflect poorer status.

$\S$ Food insecurity score was created from an eight-item household food security survey adapted from the US Household Food Security Survey Model ${ }^{(23,24)}$; food secure (0 positive responses), food insecure (1-8 positive responses). ॥ With the 'opt-in' system, women were invited to be tested for HIV; with the 'opt-out' system, all women were tested unless they specifically requested otherwise.

II lllness represented having reported at least one of the following symptoms over $30 \mathrm{~d}: \geq 3$ liquid stools/d, fever, cough or difficulty breathing. 
introduction of solids (HR 1.56;95\% CI 1.10, 2.22; $P=0 \cdot 011$ ). The association of introduction of solids with HIV-U, being of an 'other' ethnicity, and a lower WAZ all tended towards significance (HR 1.31; $95 \%$ CI 0.95, 1.81; $P=0.098$, HR $1.52 ; 95 \%$ CI $1.00,2 \cdot 31 ; P=0.052$ and HR $0.90 ; 95 \%$ CI 81, $1 \cdot 01 ; P=0.063$, respectively).

\section{Logistic regression}

Logistic regression for the likelihood to EBF for 6 months demonstrated similar results as the survival analysis. Mothers with HIV-P status were less than half as likely to complete 6 months of EBF compared with HIV-N mothers (adjusted OR (aOR) $0 \cdot 42 ; 95 \%$ CI $0 \cdot 22,0 \cdot 81 ; P=0 \cdot 010$ ). The 'other' ethnicity category had a lower odds of EBF (aOR 0.35; $95 \%$ CI 0.13, 0.93; $P=0.036)$ compared with being Ga/Adangbe, whereas having a higher number of children under 5 years of age tended to increase the odds of EBF for at least 6 months (aOR 1.35; $95 \%$ CI $0.94,1.94 ; P=0.101)$.

\section{Risk of weaning and WHO policy change}

By the end of the 1st year, a 5-6-fold higher percentage of infants were weaned in the HIV-P group compared with HIV-N and HIV-U (26 v. 4 and 5\%, respectively; $P<0.0001$; Table 2). The prevalence of weaning between 7 and 12 months was similar for infants born before or after the release of the WHO breast-feeding guidelines when analysed across the different HIV groups (all months $P>0 \cdot 10$ ). However, when analysed among only the HIV-P group, which had substantial weaning, there tended to be a difference; infants born before 2007 were twice as likely to be weaned at 12 months than those who were born in 2007 (22.7 v. 46.7\%; $P=0.051)$.

\section{Discussion}

This study provided a unique opportunity to examine EBF and the introduction of liquids and solids/semi-solids into infants' diets in an HIV-affected region of Ghana. The association of infant feeding with HIV status and other predictors varied depending on the specific behaviour that was being carried out, suggesting that multiple intervention approaches may be needed to fully promote optimal feeding.

In our study site, HIV infection was linked to being less likely to follow the international recommendation to EBF for 6 months; the duration difference associated with HIV was almost 1 month. Some studies, but not all, concur with our results. The feeding practices of a random selection of 727 mothers (HIV-U) participating in a 2003 district-wide survey were compared with those of 235 HIV-infected mothers attending outreach clinics in 2005 in the same area of Uganda $^{(29)}$. Among those with children under 6 months of age, only $24 \%$ of HIV-infected mothers reported EBF compared with $45 \%$ of the general population $(P<0.05)$. The prevalence of EBF in both groups may have been inflated, as it was estimated using a 24-h dietary recall. Infants experience brief interruptions in an EBF pattern ${ }^{(30,31)}$ that can be easily not reported in cross-sectional surveys. Twice-weekly home visits allowed our project field staff to establish rapport with the mothers and to identify precisely any changes in feeding practices. In addition, mothers' willingness to respond truthfully to questions about breast-feeding may vary by HIV status, leading to reporting bias. Increased trust between mothers and field staff established by frequent visits can be expected to improve data quality.

In contrast to our results and those in Uganda, a longitudinal study in 2001 that followed a cohort of 177 HIV-infected and 177 HIV-uninfected Zambian mothers from pregnancy to 4 months postpartum found no difference in EBF duration ${ }^{(32)}$. The 4-month EBF rate for both groups was the same as that of our HIV-P mothers (37\%); however, uninfected women in our setting had a higher rate of $51 \%$. Our overall EBF median survival time of 5.3 months was higher than national rates reported in $2003(2.3 \text { months })^{(20)}$ or $2008(3.3 \text { months })^{(18)}$. These differences may be partly because of selection bias. All participants were recruited through hospital VCT clinics where mothers were exposed to infant feeding education. In addition, the project referred mothers to an independent breast-feeding counsellor if they requested assistance. Aidam et al. ${ }^{(33)}$ demonstrated the potential for high-quality counselling to improve EBF duration. In their study carried out close to our site, almost all mothers with prenatal and postnatal lactation counselling chose to EBF for 6 months compared with $48 \%$ of those mothers who received standard-of-care health services $(P=0.008)$. Laar et $a l .{ }^{(10)}$ reported that mothers living in districts adjacent to our study site were discouraged from using formula by their HIV nurse counsellors. Healthcare providers reported that even if the appropriate circumstances were present formula was not promoted because the hospitals were signatories of the Baby Friendly Health Initiative. Their commitment to breastfeeding supports the higher EBF duration results from our population; however, the almost 1-month difference in EBF associated with HIV status suggests that there remained an inadequacy in HIV-specific feeding education and support. The dangers of mixed feeding should be emphasised to all mothers; however, the additional risk of MTCT of $\mathrm{HIV}^{(6)}$ demonstrates the need for specific 'how-to' messages and counselling for HIV-P mothers.

In addition to the influence of health services, mothers' infant feeding decisions may have been influenced by the child's health and nutritional status. Better nutrition was associated with a decreased risk of stopping EBF and introducing formula, and a tendency to not introduce non-milk liquids and solids. In other settings, weaning age was inversely related to the nutritional status and health of the child ${ }^{(34,35)}$

There are inconsistent results regarding the association between HIV and the introduction of non-breast milk items. In Lusaka, Zambia, HIV-infected mothers introduced liquids to their infants below 2 months of age more frequently than uninfected mothers ( $28 v .11 \%$, respectively; $P=0.03$ ) and only HIV-infected mothers introduced semi-solids before 2 months $(P=0.002)^{(36)}$. Women reported introducing foods early because of a perception of milk insufficiency, a common belief in our study district ${ }^{(37)}$. In the previously mentioned Ugandan study, HIV-infected mothers compared with women in the general population also reported early (before 6 months) 
introduction of water ( $54 v .23 \% ; P<0 \cdot 001)$, animal-based milks (54 v. 35\%; $P<0.05)$ and solids $(49 \text { v. } 14 \% ; P<0.001)^{(29)}$. In contrast, Ghanaian HIV-P mothers were less likely to give formula and non-human milks than other liquids.

Even with its low use, HIV status was associated with an increased risk of early introduction of animal-based milks. Women in other poor settings reported the decision to not wean specifically because of their inability to purchase milk, which they considered to be the acceptable replacement for breast milk $^{(34)}$. It is likely that similar decisions were made by our Ghanaian study population; a higher amenities score (more wealth) was associated with an increased risk of introducing milk. These results are consistent with those of Laar et al. ${ }^{(10)}$ who reported that the perceived high cost of replacement feeding was associated with an increased likelihood of EBF in the first 3 months of life in Ghana (aOR 4.60; 95\% CI 1.40, 15.14). Economic considerations need to be part of nutrition counselling.

In our study, being of an 'other' ethnic group (half of whom were northerners) was associated with the increased risk of stopping EBF. The Ga/Adangbe was the predominant ethnic group. Mothers from the local groups may have had more social networks to provide emotional and physical support, which has been found to help mothers properly bond with a new infant, use health services more successfully and ultimately to engage in better feeding practices ${ }^{(38)}$. Further understanding of the diverse cultural practices and beliefs in Ghana is needed.

The HIV-U category is primarily a reflection of the 'opt-in' HIV testing policy. When clinics changed to an 'opt-out' HIV testing policy, fewer women refused testing. Although the distribution of HIV-P and HIV-N mothers in this group is unknown, the HIV-U results here and in previous publications ${ }^{(22)}$ suggest that the group is a combination of infected and uninfected women.

Over the time of the project, the WHO changed its infant feeding guidelines related to HIV. In 2003, WHO guidelines stated '... exclusive breastfeeding is recommended during the first months of life and should then be discontinued as soon as feasible $^{,(39)}$. By mid-project in late 2006, new guidelines were developed that recommended stopping 'once a nutritionally adequate and safe diet without breast milk can be provided, ${ }^{,(28)}$. One expected to see a higher prevalence of weaning among the early study participants. However, weaning prevalence among infants in the HIV-P group tended to be higher at 12 months among those born after the new guidelines were released. Either other factors related to time were relevant or the new policy had the unintended result of increased weaning among a population that most likely did not meet the suggested conditions.

The study would have been enriched by having a qualitative component to look in-depth at unmeasured maternal and household characteristics, maternal and stakeholders' knowledge and beliefs regarding feeding practices, and social dynamics in the home, health setting and community. Furthermore, the relatively small sample size and the large number of censored cases for some foods may have diminished the ability to identify meaningful associations. Although recall bias may have existed because of mothers' self-reporting of feeding practices, this was minimised by collecting information twice weekly.
The associations between HIV and infant feeding practices reported in this paper are likely to be as relevant today as they were 10 years ago. National-level economic and health improvements over the past 10 years have not occurred homogeneously. Although the country met the Millennium Development Goal of halving extreme poverty, the Eastern Region experienced an increase in poverty incidence between 2006 and $2013^{(40)}$. As the nation experienced a $64 \%$ decrease in the prevalence of HIV between 2007 and 2013, the Eastern region's rate increased and remains today the highest in the country at $3.7 \%$, the same as $2003^{(41)}$. Experiences of stigma in health services ${ }^{(42)}$ and an unstable supply of antiretroviral drugs remain problematic. Although general breast-feeding promotion is well established in the Ghana Health Services, interactive education is needed to provide practical solutions to problems that mothers have in practicing EBF and complementary feeding. Recent research has documented little improvement in the quality of the nutrition education in rural communities of the Eastern region ${ }^{(10)}$

This study investigated the association between HIV status and infant feeding practices in Ghana. EBF is the most effective and feasible feeding option for mothers in low-resource settings; increased and better interventions are needed to reach the WHO's 2025 goal and to ensure that all women receive the support needed. New strategies will require inter-sector and multi-level approaches that include strong coordination between facility and community-based infant feeding promotion, protection and support systems.

\section{Acknowledgements}

The authors would like to thank Boateng Bannerman and Rula Soueida for their work on data management and Dr Roger Cue for assistance with the statistical analyses. The authors are indebted to the research and health services teams that worked with us and the families that let us into their lives.

This work was supported by the National Institute of Child Health and Development (NICHD)/National Institutes of Health (grant no. HD 43260). The National Institutes of Health had no role in the design, analysis or writing of this article. The contents are solely the responsibility of the authors and do not necessarily represent the official views of NICHD.

G. S. M., A. L., R. P.-E., R. E. M. and L. B. developed the research questions, designed the study and participated in the field work; K. A. B. carried out the statistical analysis; G. S. M. and K. A. B. drafted the paper; A. L., R. P.-E., R. E. M. and L. B. contributed to the writing of the paper; all authors contributed to the interpretation of results.

There are no conflicts of interest.

\section{References}

1. Joint United Nations Programme on HIV/AIDS (2013) Global report: UNAIDS report on the global AIDS epidemic 2013. http:// $\lambda$.unaids.org/sites/default/files/media_asset/UNAIDS_ Global_Report_2013_en_1.pdf (accessed August 2015).

2. Black RE, Victora CG, Walker SP, et al. (2013) Maternal and child undernutrition and overweight in low-income and middle-income countries. Lancet 382, 427-451. 
3. Bartick M \& Reinhold A (2010) The burden of suboptimal breastfeeding in the United States: a pediatric cost analysis. Pediatrics 125, e1048-e1056.

4. Coutsoudis A, Pillay K, Kuhn L, et al. (2001) Method of feeding and transmission of HIV-1 from mothers to children by 15 months of age: prospective cohort study from Durban, South Africa. AIDS 15, 379-387.

5. Coovadia HM, Rollins NC, Bland RM, et al. (2007) Motherto-child transmission of HIV-1 infection during exclusive breastfeeding in the first 6 months of life: an intervention cohort study. Lancet 369, 1107-1116.

6. Iliff PJ, Piwoz EG, Tavengwa NV, et al. (2005) Early exclusive breastfeeding reduces the risk of postnatal HIV-1 transmission and increases HIV-free survival. AIDS 19, 699-708.

7. World Health Organization (2010) Guidelines on HIV and infant feeding 2010: principles and recommendations for infant feeding in the context of HIV and a summary of evidence. http://apps.who.int/iris/bitstream/10665/44345/ 1/9789241599535_eng.pdf (accessed August 2015).

8. Cai X, Wardlaw T \& Brown DW (2012) Global trends in exclusive breastfeeding. Int Breastfeed J 7, 12.

9. World Health Organization (2014) Global Nutrition Targets 2025: breastfeeding policy brief. http://apps.who.int/iris/ bitstream/10665/149022/1/WHO_NMH_NHD_14.7_eng.pdf? ua $=1$ (accessed August 2015).

10. Laar A, Ampofo W, Tuakli JM, et al. (2009) Infant feeding choices and experiences of HIV-positive mothers from two Ghanaian districts. J AIDS HIV Res 1, 23-33.

11. Kakute P, Ngum J, Mitchell P, et al. (2005) Cultural barriers to exclusive breastfeeding by mothers in a rural area of Cameroon, Africa. J Midwifery Womens Health 50, 324-328.

12. Bland R, Rollins NC, Coutsouis A, et al. (2002) Breastfeeding practices in an area of high HIV prevalence in rural South Africa. Acta Paediatr 91, 704-711.

13. Fjeld E, Siziya S, Katepa-Bwalya M, et al. (2008) 'No sister, the breast alone is not enough for my baby' a qualitative assessment of potentials and barriers in the promotion of exclusive breastfeeding in southern Zambia. Int Breastfeed J 3, 26.

14. Sibeko L, Dhansay MA, Charlton KE, et al. (2005) Beliefs, attitudes, and practices of breastfeeding mothers from a periurban community in South Africa. J Hum Lact 21, 31-38.

15. Doherty T, Chopra M, Nkonki L, et al. (2006) Effect of the HIV epidemic on infant feeding in South Africa: 'When they see me coming with the tins they laugh at me'. Bull World Health Organ 84, 90-96.

16. World Health Organization (2012) Resolution WHA65.6. Comprehensive Implementation Plan on Maternal, Infant and Young Child Nutrition. Resolutions and decisions, annexes. Sixty-Fifth World Health Assembly, Geneva, 21-26 May. http:// www.who.int/nutrition/ topics/WHA65.6_resolution_en.pdf? ua $=1$ (accessed August 2015)

17. Ghana AIDS Commission (2014) Country aids response progress report - Ghana. Reporting period January 2013December 2014. http://www.unaids.org/sites/default/files/ country/documents//GHA_narrative_report_2014.pdf (accessed August 2015).

18. Ghana Statistical Service, Ghana Health Service \& ICF Macro (2009) Ghana Demographic and Health Survey 2008. Accra: GSS, GHS \& ICF Macro.

19. Ghana Statistical Service, Ghana Health Service \& The Demographic and Health Surveys Program ICF International (2015) Ghana Demographic and Health Survey 2014 key indicators. http://dhsprogram.com/pubs/pdf/PR59/PR59.pdf (accessed August 2015).

20. Ghana Statistical Service, Noguchi Memorial Institue for Medical Research \& ORC Macro (2004) Ghana Demographic and Health Survey 2003. Calverton, MD: GSS, NMIMR \& ORC Macro.

21. Okronipa HET, Marquis GS, Lartey A, et al. (2012) Postnatal depression symptoms are associated with increased diarrhea among infants of HIV-positive Ghanaian mothers. AIDS Behav 16, 2216-2225.

22. Lartey A, Marquis GS, Mazur R, et al. (2014) Maternal HIV is associated with reduced growth in the first year of life among infants in the eastern region of Ghana: the Research to Improve Infant Nutrition and Growth (RIING) project. Matern Child Nutr 10, 604-616.

23. Garcia J, Hromi-Fiedler A, Mazur RE, et al. (2013) Persistent household food insecurity, HIV, and maternal stress in periurban Ghana. BMC Public Health 13, 215.

24. National Research Council (2006) Survey measurement of food insecurity and hunger. In Food Insecurity and Hunger in the United States: An Assessment of the Measure. Panel to Review the US Department of Agriculture's Measurement of Food Insecurity and Hunger, pp. 55-70 [GS Wunderlich \& JL Norwood, editors]. Washington, DC: The National Academies Press.

25. Cox JL, Holden JM \& Sagovsky R (1987) Detection of postnatal depression. Development of the 10-item Edinburgh Postnatal depression scale. Br J Psychiatry 150, 782-786.

26. World Health Organization (1991) Indicators for Assessing Breastfeeding Practices. Geneva: WHO. http://apps.who. int/iris/bitstream/10665/62134/1/WHO_CDD_SER_91.14.pdf (accessed August 2015).

27. World Health Organization Multicenter Growth Reference Study Group (2006) WHO Child Growth Standards: Length/ Height-for-Age, Weight-for-Age, Weight-for-Length, Weightfor-Height And Body Mass Index-for-Age: Methods and Development. Geneva: WHO.

28. World Health Organization, United Nations International Children's Emergency Fund \& Joint United Nations Programme on HIV/AIDS (2006) HIV and infant feeding update based on the technical consultation held on behalf of the Inter-Agency Tast Team (IATT) on prevention of HIV infection in pregnant women, mothers and their infants, Geneva, 25-27 October. http://apps.who.int/iris/bitstream/10665/43747/1/ 9789241595964_eng.pdf (accessed December 2015).

29. Fadnes LT, Engebretsen IMS, Wamani H, et al. (2009) Infant feeding among HIV-positive mothers and the general population mothers: comparison of two cross-sectional surveys in Eastern Uganda. BMC Public Health 9, 124.

30. Marquis GS, Díaz J, Bartolini R, et al. (1998) Recognizing the reversible nature of child-feeding decisions. Breastfeeding, weaning, and relactation patterns in a shanty town community of Lima, Peru. Soc Sci Med 47, 645-656.

31. Zohoori N, Popkin BM \& Fernandez ME (1993) Breast-feeding patterns in the Philippines: a prospective analysis. J Biosoc Sci 25, 127-138.

32. Chisenga M, Kasonka L, Makasa M, et al. (2005) Factors affecting the duration of exclusive breastfeeding among HIV-infected and-uninfected women in Lusaka, Zambia. J Hum Lact 21, 266-275.

33. Aidam BA, Pérez-Escamilla R \& Lartey A (2005) Lactation counseling increases exclusive breast-feeding rates in Ghana. J Nutr 135, 1691-1695.

34. Marquis GS, Habicht JP, Lanata CF, et al. (1997) Association of breastfeeding and stunting in Peruvian toddlers: an example of reverse causality. Int $J$ Epidemiol 26, 349-356.

35. Simondon KB, Simondon F, Costes R, et al. (2001) Breastfeeding is associated with improved growth in length, but not weight, in rural Senegalese toddlers. Am J Clin Nutr 73, 959-967. 
36. Omari AAA, Luo C, Kankasa C, et al. (2003) Infant-feeding practices of mothers of known HIV status in Lusaka, Zambia. Health Policy Plann 18, 156-162.

37. Otoo GE, Lartey AA \& Pérez-Escamilla R (2009) Perceived incentives and barriers to exclusive breastfeeding among periurban Ghanaian women. J Hum Lact 25, 34-41.

38. Webb-Girard A, Cherobon A, Mbugua S, et al. (2012) Food insecurity is associated with attitudes towards exclusive breastfeeding among women in urban Kenya. Matern Child Nutr 8, 199-214.

39. World Health Organization, United Nations International Children's Emergency Fund \& Joint United Nations Programme on HIV/AIDS (2003) Guidelines for Decision-Makers - A Guide for Health Care Managers and Supervisors. Geneva: WHO.
40. National Development Planning Commission (2015) Ghana millennium development goals 2015 report. http://www. undp.org/content/dam/ghana/docs/Doc/Inclgro/UNDP_GH_ 2015\%20Ghana\%20MDGs\%20Report.pdf (accessed December 2015).

41. Ghana AIDS Commission (2015) Country AIDS response progress report - Ghana reporting period January 2013December 2014. http://www.unaids.org/sites/default/files/ country/documents//file,94605,es..pdf (accessed December 2015).

42. Dako-Gyeke M, Dako-Gyeke P \& Asampong E (2015) Experiences of stigmatization and discrimination in accessing health services: voices of person living with HIV in Ghana. Soc Work Health Care 54, 269-285. 